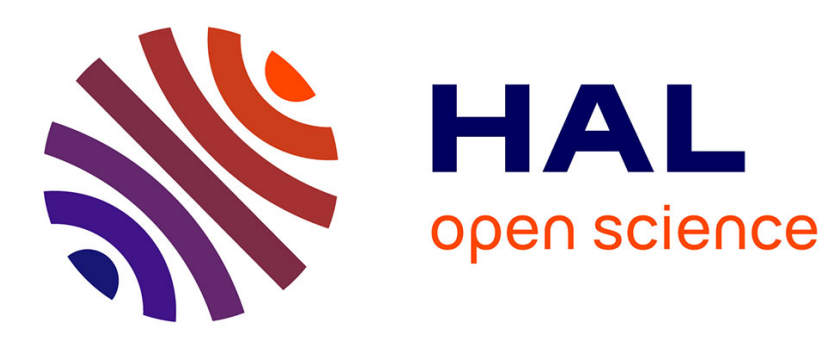

\title{
TROUBLES DU GENRE ET DE LA SEXUALITÉ: CONFLITS ET CONSENSUS
}

\author{
Julie Mazaleigue-Labaste
}

\section{To cite this version:}

Julie Mazaleigue-Labaste. TROUBLES DU GENRE ET DE LA SEXUALITÉ: CONFLITS ET CONSENSUS. L'Information Psychiatrique, 2011, 87 (4), pp.305. 10.1684/ipe.2011.0776 . halshs01989304

\section{HAL Id: halshs-01989304 https://shs.hal.science/halshs-01989304}

Submitted on 25 Jan 2019

HAL is a multi-disciplinary open access archive for the deposit and dissemination of scientific research documents, whether they are published or not. The documents may come from teaching and research institutions in France or abroad, or from public or private research centers.
L'archive ouverte pluridisciplinaire HAL, est destinée au dépôt et à la diffusion de documents scientifiques de niveau recherche, publiés ou non, émanant des établissements d'enseignement et de recherche français ou étrangers, des laboratoires publics ou privés. 


\section{TROUBLES DU GENRE ET DE LA SEXUALITÉ : CONFLITS ET CONSENSUS}

Julie Mazaleigue-Labaste

John Libbey Eurotext | «L'information psychiatrique»

2011/4 Volume 87 | pages 305 à 312

ISSN 0020-0204

Article disponible en ligne à l'adresse :

https://www.cairn.info/revue-l-information-psychiatrique-2011-4-page-305.htm

Distribution électronique Cairn.info pour John Libbey Eurotext.

(C) John Libbey Eurotext. Tous droits réservés pour tous pays.

La reproduction ou représentation de cet article, notamment par photocopie, n'est autorisée que dans les limites des conditions générales d'utilisation du site ou, le cas échéant, des conditions générales de la licence souscrite par votre établissement. Toute autre reproduction ou représentation, en tout ou partie, sous quelque forme et de quelque manière que ce soit, est interdite sauf accord préalable et écrit de l'éditeur, en dehors des cas prévus par la législation en vigueur en France. Il est précisé que son stockage dans une base de données est également interdit. 


\title{
TROUBLES LIÉS AU GENRE
}

\section{Troubles du genre et de la sexualité : conflits et consensus}

\author{
Julie Mazaleigue-Labaste
}

\begin{abstract}
RÉSUMÉ
Ce rapport analytique des interventions et des discussions de la journée d'études du 17 décembre 2010 résume les problématiques abordées, les points de conflits et les consensus qui ont pu se dégager au sujet de la révision des catégories « Troubles de l'identité sexuelle », codée en F64, et « Troubles de la préférence sexuelle », codée en F65, de la 10e classification internationale des maladies, dans la perspective de réélaboration de cette dernière (CIM-10 vers CIM-11).
\end{abstract}

Mots clés : identité sexuée, sexualité, transsexualisme, classification, cim, stigmatisation

\begin{abstract}
Gender identity and sexuality disorders: debates and consensus. This report describes problems, points of conflicts, and consensus which have emerged from communications and debates about the revision of two categories of ICD-10, "gender identity disorder" (diagnosis code F64) and "paraphilias" (diagnosis code F65), during the December, $17^{\text {th }} 2010$ seminar dedicated to the relations between psychiatric classifications and stigmatization processes.
\end{abstract}

Key words: sexual identity, sexuality, transsexualism, classification, icd, stigmatization

\section{RESUMEN}

Trastornos de género y sexualidad : debates y consensos. Este informe analítico de las intervenciones y discusiones de la jornada de trabajo del 17 de diciembre de 2010 resume las problemáticas abordadas, los puntos conflictivos y los consensos que han podido despejarse sobre la revisión de las categorías de los "Trastornos de la identidad sexual”, codificada como F64, y las de los "Trastornos de la orientación sexual", codificada como F65, de la décima Clasificación Internacional de las Enfermedades, en la perspectiva de reelaborar la misma (CIM-10 hacia CIM-11).

Palabras claves : identidad sexuada, sexualidad, transexualismo, clasificación, cim, estigmatización

\section{Introduction}

Le présent rapport est un résumé analytique des interventions et des discussions de la journée, des problématiques abordées, des points de conflits et des consensus qui ont pu se dégager au sujet de la révision des catégories « Troubles

Docteur en philosophie, agrégée de philosophie, ATER Université de Picardie Jules-Verne, Centre d'histoire des sciences, des sociétés et des conflits, UPJV, Amiens, France

<julie.mazaleigue@wanadoo.fr>

Tirés à part : J. Mazaleigue-Labaste de l'identité sexuelle », codée en F64, et « Troubles de la préférence sexuelle », codée en F65, dans le chapitre V (« Troubles mentaux et du comportement ») de la $10^{\mathrm{e}}$ classification internationale des maladies (CIM-10). Il se clôt sur le rappel des propositions de modification qui ont été soumises ${ }^{1}$.

Cette troisième journée d'études ${ }^{2}$ portant sur les relations entre classifications et stigmatisation a affiché l'ambition de contribuer à la dynamique de réélaboration de la CIM en construisant un espace de dialogue entre

\footnotetext{
1 Section 5 .

${ }^{2}$ Les deux premières ayant respectivement porté sur les psychoses et la psychiatrie infanto-juvénile.
} 
différents acteurs. Trois types de points de vue s'y sont croisés $^{3}$. Premièrement, celui des professionnels de la santé mentale, psychiatres pour la plus grande part ${ }^{4}$. Deuxièmement, celui des associations trans' et des usagers ${ }^{5}$. Troisièmement, le point de vue analytique des chercheurs en sciences sociales ${ }^{6}$. Cette distinction entre ces trois catégories de sujets doit néanmoins être relativisée, car les représentants des associations possèdent aussi une expertise psy et mobilisent activement les travaux des sciences sociales sur le sexe et le genre ${ }^{7}$. Cette impossibilité du maintien des relations et des hiérarchies traditionnelles entre les différents acteurs est analysée dans le premier temps du présent rapport.

L'objet principal des communications et des discussions de la journée fut la catégorie $\mathrm{F} 64^{8}$, objet de six interventions sur les sept présentées le 17 décembre 2011. D'un point de vue quantitatif, les troubles de la préférence sexuelle ont donc reçu un traitement marginal, ce qui explique la place prise dans ce rapport par la catégorie F64. L'objet central de cette journée fut l'articulation entre classifications, processus de stigmatisation, et revendications politiques. Au problème des enjeux politiques de l'expertise (section 1), s'est adjointe une interrogation sur les fonctions des classifications (section 2). Si certains des problèmes soulevés par F65 sont communs avec les difficultés posées par les « troubles de l'identité sexuelle », ils sont cependant le lieu d'interrogations qui leur sont propres, notamment en relation à la sphère médico-judiciaire, ce qui a conduit à leur réserver un résumé séparé (section 3 ). Il est suivi d'une mise en perspective critique de l'ensemble des analyses (section 4).

Il est essentiel d'indiquer le déplacement opéré, d'un paradigme du trouble mental - la pathologie, pensée en termes négatifs - à un modèle positif de la santé mentale, et plus largement de la santé sexuelle ${ }^{9}$. Ces enjeux dépassent

\footnotetext{
${ }^{3}$ Comme l'a souligné Jean-Luc Roelandt dans la présentation de la journée.

4 Jean-Luc Roelandt, Colette Chiland, Tiphaine Séguret, et Pierre Hum pour les intervenants. Des responsables de la Société francaise d'etudes et de prise en charge du transsexualisme (SoFECT) ont aussi été invités.

5 Tom Reucher, psychologue clinicien spécialisé dans les questions de transidentité, et représentant des associations d'usagers ; Vincent Guillot, ancien président Europe de l'Organisation internationale des intersexes (OII) ; Maud Thomas, cofondatrice et présidente de l'association Sans Contrefaçon. On reviendra sur les deux termes « trans' » et « usagers ». Le choix d'utiliser le terme «trans' », employé par tous, est volontaire, et repose sur les nombreuses critiques adressées aux différentes catégories, décrites plus bas.

${ }^{6}$ Anne Lovell, Alain Giami, Eric Macé.

${ }^{7}$ Tom Reucher est psychologue clinicien, Maud Thomas mène des recherches en anthropologie du(des) genre(s). De plus, les théories des sciences sociales, et plus particulièrement des social studies, des gender studies, et des théories queer, contribuent au discours des associations.

${ }^{8}$ Dans ce groupe, la catégorie de «transsexualisme » (F64.0) a constitué la cible principale des réflexions et des critiques.

${ }^{9}$ Alain Giami, Patrice Desmons, Tom Reucher, Maud Thomas, et Geoffrey M. Reed, chef de mission pour la révision de la CIM-10 (Santé mentale et
}

largement le domaine de la psychopathologie et se posent en termes éthiques, politiques, et juridiques internationaux (droits humains). Le renversement est crucial : ce ne sont pas simplement les raisons cliniques, mais aussi les raisons de santé, pensée comme une structure holistique ${ }^{10}$ relevant d'un droit positif, qui doivent guider l'élaboration des catégories adéquates pour le diagnostic et le suivi médical. À ce titre, le rapport de Thomas Hammarberg ${ }^{11}$ sur les droits de l'homme et l'identité de genre ${ }^{12}$ est incontournable pour cette révision des classifications qui ne peut faire l'économie du problème des discriminations ${ }^{13}$. La prise en compte de ce point de vue des droits humains exige alors une réflexion et des propositions proportionnées à la valeur transnationale de la CIM et à son usage par des non psychiatres. La fonction des classifications dans les processus de stigmatisation doit ainsi être repensée à l'échelle d'un contexte mondial, en prenant en compte que dans nombre de pays, ce sont en premier lieu l'absence de diagnostic, de soin, et les violations graves des droits humains qui constituent les problèmes urgents.

Au cours de cette journée se sont exprimés un certain nombre de conflits entre les différents points de vue, notamment entre celui des psychiatres de la transsexualité et celui des associations. Toutefois, une forme de consensus semble avoir été atteinte en fin de journée quant à la révision de F64, à minima sur les perspectives générales et de travail à adopter. Si elle n'a pas conduit à l'élaboration d'une proposition précise comme l'exige l'OMS, cette journée n'en a pas moins permis de poser les jalons d'une construction commune aux professionnels et aux usagers, d'un savoir autre et d'un savoir ensemble ${ }^{14}$.

\section{Les enjeux politiques de l'expertise}

La place, le pouvoir et le point de vue des sujets classifiés dans la révision des classifications ont été prépondérants ${ }^{15}$. Il est difficile de maintenir la traditionnelle différence entre les professionnels et les usagers, qui repose implicitement

\footnotetext{
troubles du comportement), Département de la santé mentale et de l'abus des substances, OMS, Genève.

${ }^{10}$ Conformément à la définition de la santé promue par l'OMS : «La santé est un état de complet bien-être physique, mental et social, et ne consiste pas seulement en une absence de maladie ou d'infirmité ».

${ }^{11}$ Haut commissaire aux droits de l'homme du Conseil de l'Europe

${ }^{12}$ Hammarberg T. Human Rights and Gender Identity. CommDH/ IssuePaper, 2009 (2), Strasbourg : Office of the commissionner of human rights, Council of Europe, 29 Juillet 2009, 53 pages.

https://wcd.coe.int/wcd/com.instranet.InstraServlet?Index=no\&command $=$ com. instranet. CmdBlobGet $\&$ InstranetImage $=1670860 \&$ SecMode $=1 \&$ DocId $=1433126 \&$ Usage $=2$

Version française disponible :

https://wcd.coe.int/wcd/com.instranet.InstraServlet?Index=no\&command $=$ com .instranet CmdBlobGet $\&$ InstranetImage $=1692292 \&$ SecMode $=1 \&$ DocId $=1458356 \&$ Usage $=2$

13 Comme l'a rappelé Geoffrey Reed.

${ }^{14}$ Patrice Desmons, Jean-Luc Roelandt.

15 Jean-Luc Roelandt, Patrice Desmons, Alain Giami.
} 
sur une hiérarchie et une asymétrie conceptuelle savants/profanes ${ }^{16}$. Les représentants des associations ${ }^{17}$ ont rappelé que leur expérience fonctionnait comme expertise, se développant aussi dans le champ psy ${ }^{18}$. C'est à l'aune de ce brouillage des frontières, symptomatique de leur déplacement, qu'il faut lire le rejet exprimé par ces représentants ${ }^{19}$ du terme d' « usager », qui marque le refus du maintien des sujets classifiés à l'extérieur du débat.

La relation des professionnels de santé mentale à la fonction de l'expertise est ainsi à interroger ${ }^{20}$. La revendication d'une expertise par les médecins implique en effet, par définition, une forme de confiscation du savoir et de dénégation de la valeur épistémique de la parole de l'autre, fixant l'ordre du discours et la hiérarchisation des sujets parlants en fonction du partage savant/profane ${ }^{21}$. Cette revendication d'une expertise ne peut alors que mener au conflit des expertises, chacun de part et d'autre se réclamant d'un savoir exclusif, ce qui mine par principe toute possibilité de collaboration. Peut-on, au contraire, penser que la fonction des professionnels de santé mentale est avant tout clinique, impliquant le diagnostic différentiel et l'accompagnement, sans dépasser ces limites ? Cette question doit être adressée aux classifications elles-mêmes : dans quelle mesure peuvent-elles, et doivent-elles, rester des outils cliniques ?

Cette question délicate de la place et de la fonction de l'expertise est aussi posée par la classification et la prise en charge des troubles de la préférence sexuelle. Tiphaine Séguret a ainsi souligné les attentes démesurées en termes d'expertise qu'adresse le système judiciaire aux psychiatres dans les cas de délinquance et de criminalité sexuelles, qui menacent les fonctions diagnostiques et thérapeutiques. Qu'il s'agisse des questions trans' ou des cas tombant sous la catégorie F65, les problèmes sont analogues : l'effacement de la fonction de soignant derrière celle d'expert ${ }^{22}$.

Quant aux «troubles de l'identité sexuelle », l'existence de réels points de dissensions et de conflits n'a nullement été minorée par les acteurs. Les psychiatres et les associations font consensus sur un constat : le dialogue est éminemment difficile, et il existe une forte suspicion mutuelle. Les médecins se sentent ainsi attaqués par les militants, et les trans' stigmatisés par les médecins et dépossédés de leur expérience. Deux constats symétriques ont ainsi été formulés. Premièrement, en dépit des appels à l'échange affichés de part et d'autre, les confrontations persistent,

\footnotetext{
16 Alain Giami.

${ }^{17}$ Maud Thomas, Tom Reucher, Vincent Guillot.

18 Comme l'a rappelé Patrick Desmons.

${ }^{19}$ Maud Thomas, Vincent Guillot.

${ }^{20}$ Patrice Desmons, Tiphaine Séguret.

${ }^{21}$ Expertise qu'a clairement revendiquée Mireille Bonierbale, président de la SoFECT, face à Tom Reucher.

22 Une différence est néanmoins à souligner : dans le cas des troubles de la préférence sexuelle, ce sont les psychiatres eux-mêmes qui sont encombrés par leur tâche.
}

tout comme les évitements mutuels ${ }^{23}$. Deuxièmement, les représentants du corps médical ${ }^{24}$ ont déploré que certains usagers non militants ne soient pas représentés, et les représentants des associations ${ }^{25}$ ont regretté que les professionnels de santé rencontrés dans le cadre associatif soient absents du débat du 17 décembre. Ce second constat signifie que les espaces de rencontre existent, et que le dialogue est possible. Il est cependant loin d'aller de soi. Les représentants des associations ont souligné que ces difficultés étaient les corollaires de la catégorisation des trans' comme atteints d'un « trouble mental », et appartenaient au processus de stigmatisation. Elles ne sont toutefois pas seulement liées aux classifications et aux diagnostics, mais aussi aux pratiques, notamment aux restrictions dans le choix des équipes médicales ${ }^{26}$. Le conflit ne siège donc pas, en général, entre « le pouvoir médical » et les « usagers », mais se cristallise très précisément entre les associations et les équipes hospitalières historiques représentées par la SoFECT, les premières dénonçant une mainmise institutionnelle des dernières sur l'ensemble des protocoles de soin ${ }^{27}$. À ce qui est vécu comme une confiscation, les associations opposent la revendication d'une liberté de choix des médecins et des parcours de $\operatorname{soin}^{28}$.

Les conflits sont donc déplorés de part et d'autre. Cette journée a constitué un moyen d'objectiver et de penser ces confrontations, et d'esquisser une dynamique de collaboration, essentielle, comme l'a rappelé Geoffrey Reed, pour la révision de la CIM-10. Dans la perspective du respect des droits humains, la prise en compte des acteurs trans' est incontournable.

\section{Les fonctions des classifications}

Si le fait que les classifications jouent un rôle dans les processus de stigmatisation et la nécessité de réviser F64 et F65 sont objets de consensus, l'accord ne s'est pas établi sur les fonctions précises des catégories dans ce processus, ni sur l'articulation entre catégories et stigmatisation. Quelles sont les fonctions respectives des classifications savantes, des pratiques médicales, des opinions communes, et des catégories populaires ${ }^{29}$ ? En ce cas, sur quoi la révi-

\footnotetext{
23 Colette Chiland a ainsi déploré la difficulté des échanges avec les associations, tandis que Tom Reucher a souligné les tentatives de dialogue menées par les associations face à la SoFECT depuis 20 ans, en rappelant l'absence d'intervenants et d'experts trans' dans les conférences médicales qui leur sont dédiées. Maud Thomas a dressé un état des lieux semblable. ${ }^{24}$ Colette Chiland

25 Maud Thomas.

26 Tom Reucher, Vincent Guillot.

27 Tom Reucher, Maud Thomas, Vincent Guillot.

${ }^{28}$ On trouvera une analyse très précise de ces difficultés pratiques et institutionnelles, et des alternatives proposées par les usagers dans le texte de Tom Reucher.

${ }^{29}$ La question a été posée par le philosophe, historien des sciences, psychologie clinicien et psychanalyste Pierre-Henri Castel.
} 
sion doit-elle porter : sur le contenu de F64, ou sur F64 elle-même, qui devrait être démembrée pour conduire à la dépsychiatrisation des identités trans', comme le souhaitent les associations?

\section{Quelles catégories?}

Si un étayage scientifique des classifications a été légitimement réclamé par les médecins, cette demande soulève immédiatement une difficulté de taille : il n'y a pas d'accord, ni entre associations et psychiatres, ni même à l'intérieur du monde médical, sur les catégories qui pourraient venir remplacer celles de F64 - et en particulier l'actuelle F64.0 («transsexualisme ») - et de F65. La CIM possédant une fonction diagnostique censée étayer la thérapeutique, le problème est complexe : si la classification est inadéquate, sur quoi s'appuie-t-on pour soigner, et que soigne-t-on $?^{30}$

Une chose semble a minima inéluctable : l'abandon du modèle classique de la transsexualité. Cette catégorie est contestée comme un modèle historiquement daté ${ }^{31}$, comme une construction médicale possédant des effets de distorsion importants sur les conditions trans ${ }^{\prime 32}$, inadéquate à la diversité des situations réelles ${ }^{33}$. La dépsychiatrisation, voire la dépathologisation, est un processus en cours ${ }^{34}$. Cependant, la double question de savoir quelle classe va se substituer aux actuels «troubles de l'identité sexuelle», et s'il faut la maintenir dans le chapitre V de la CIM n'a pas été réglée. Se pose alors la question de l'articulation entre le Diagnostic and Statistical Manual of Mental Disorders (DSM) en cours de réélaboration et la CIM. Deux difficultés font néanmoins obstacle à une telle comparaison. Premièrement, les contextes d'élaboration du DSM et de la CIM sont différents ${ }^{35}$. Dans le DSM, les questions trans' ont été traitées dans un contexte américain bien spécifique quant aux questions de genre. Est-il possible d'opérer une simple «traduction » d'une classification à l'autre ? On peut cependant objecter que la CIM devant avoir valeur internationale, il s'agit justement de dépasser les spécificités du cas français. Deuxièmement, les deux classifications pos-

\footnotetext{
${ }^{30}$ C'est ce qu'a souligné Tiphaine Séguret à propos de F65.

31 Alain Giami, Éric Macé.

32 De nombreuses études historiques et épistémologiques ont mis en évidence la manière dont les patients ont répondu au modèle de comportement qu'attendaient les médecins pour obtenir un accès aux soins qui dépendait de cette correspondance entre les individus et ce modèle transsexuel. Pour une analyse de détail en français, voir Castel PH. La métamorphose impensable. Essai sur le transsexualisme et l'identité personnelle. Paris : Gallimard, 2003, $551 \mathrm{p}$.

${ }^{33}$ Diversité bien mise en évidence par l'étude d'Alain Giami, et soulignée par Maud Thomas. C'est pour cette raison que Tom Reucher a choisi de substituer le terme «transidentité » à la «transsexualité ».

${ }^{34} \mathrm{Ce}$ qu'ont indiqué les analyses d'Éric Macé et d'Alain Giami, qui ont souligné le manque d'évidence des limites entre le normal et le pathologique concernant les variations de genre, ainsi que celles d'Alain Giami dédiées à la révision actuelle du DSM IV-R.

35 Maud Thomas.
}

sèdent des extensions et des finalités différentes ${ }^{36}:$ tandis que le DSM est une classification exclusivement psychiatrique à l'usage des professionnels de santé mentale, la CIM concerne toutes les maladies et doit pouvoir être employée par l'ensemble des professionnels de santé. Dans le cadre de la CIM, la dépsychiatrisation (sortie du chapitre V) n'est donc pas synonyme de démédicalisation et de perte d'accès au remboursement des soins médicaux, puisqu'il est tout à fait envisageable de déplacer les «troubles de l'identité sexuelle » reconfigurés dans un autre chapitre, voire de créer un chapitre dédié.

Si l'on peut s'interroger sur les possibilités ouvertes par la révision du DSM, force est de reconnaître que, du côté de l'American Psychiatric Association (APA) un consensus n'est toujours pas atteint sur le remplacement des « troubles d'identité de genre » de l'actuel DSM IV-R ${ }^{37}$. Plus largement, on peut, à la suite de Colette Chiland, se demander si, quelle que soit la catégorie retenue, cette dernière ne possèdera pas, en tant qu'indiquant une pathologie, des effets de discriminants et stigmatisants. Les associations répondent plus précisément à cette question, en soulignant que l'origine de la stigmatisation est d'abord la qualification des « troubles de l'identité sexuelle » comme des maladies mentales.

\section{Stigmatisation et processus de déstigmatisation}

La question de la stigmatisation rejoint celle, plus large, de la discrimination. Elle engage alors des réflexions juridiques sur la question des droits humains et déplace le problème de la caractérisation psychologique des identités trans' par les catégories aux effets générés par ces catégories. Décrire la fonction précise des classifications dans le processus de stigmatisation est donc essentiel. Cette question n'a toutefois pas reçu de réponse univoque au cours de la journée. Trois sujets sont concernés par sa résolution : les sujets savants, les trans', et « les autres », c'est-à-dire l'entourage social et familial. Quelle est la source de la stigmatisation? Quels sont les sujets stigmatisants ?

L'existence d'une stigmatisation d'origine familiale et sociale fait consensus, tout comme la nécessité subséquente d'un travail d'information et d'éducation ${ }^{38}$ visant à transformer les mentalités. Il faut cependant souligner que les analyses sur ce sujet divergent, même entre les représentants des associations. Si Colette Chiland a rappelé les réactions sociales problématiques face aux trans', en accord en cela avec Maud Thomas qui a souligné l'intolérable et même le danger inhérent à la situation de nombre de personnes trans' dans le contexte familial et social, Vincent Guillot a, à l'inverse, attiré l'attention sur l'acceptation de plus en plus

\footnotetext{
${ }^{36}$ Geoffrey Reed.

37 Alain Giami.

${ }^{38}$ Nécessité rappelée et rappelée tant par Colette Chiland que par Tom Reucher.
} 
forte des trans', en affirmant que la stigmatisation provenait bien davantage des médecins que de la population. Il serait important de s'interroger sur l'origine de ces différences de vécu et d'analyse.

Les conceptions divergentes du rôle des catégories médicales dans cette stigmatisation dépendent, in fine, de la définition de la condition trans' et de la perception que s'en font les différents acteurs ${ }^{39}$ : problème psychopathologique per $\mathrm{se}^{40}$, ou condition non pathologique, accompagnée de difficultés psychosociales qui ne sont dues qu'aux effets de réception sociale ${ }^{41}$. Dans les deux cas, c'est le statut de la souffrance qui est problématisé : est-elle inhérente à la condition trans', pouvant alors fonctionner comme un critère clinique pour les psychiatres ${ }^{42}$, ce qui a pour conséquence d'exclure du parcours de soin les individus non souffrants ${ }^{43}$ en érigeant la douleur en norme, ou estelle l'effet psychologique de la stigmatisation sociale des identités trans' ?44

La fonction des classifications dans le processus de stigmatisation ne fait donc pas consensus. Il y a clivage entre l'appréhension médicale des catégories, perçues comme nécessaires pour la clinique et le diagnostic différentiel ${ }^{45}$, et celle des associations, qui soulignent leur caractère en soi stigmatisant. Le principal problème n'est pas l'existence d'une classification médicale incluant les identités trans', mais bien d'une catégorisation psychiatrique, renvoyant les individus à un être de « malade mental ». La performativité ${ }^{46}$ de toute classification, ne se limitant pas à décrire la réalité mais assignant de force des identités qui sont à la source de la stigmatisation, est ainsi en question. En ce point, les difficultés propres aux personnes trans' rejoignent la « fabrication générale de stigmatisation dans la société ${ }^{47}$ par les classifications psychiatriques.

La demande de dépsychiatrisation formulée par les associations ne correspond donc pas à une demande de démédicalisation - qui rendrait impossible la prise en charge des soins hormonaux et/ou chirurgicaux - ni au refus de toute prise en charge psychologique ${ }^{48}$, la santé mentale et sexuelle restant au cœur des préoccupations des trans'. Le problème économique de remboursement des soins par l'Assurance maladie, qui repose sur le constat d'affection de longue durée (ALD) impose une approche pragmatique

\footnotetext{
${ }^{39}$ Castel, op. cit.

${ }^{40}$ Colette Chiland.

${ }^{41}$ Point de vue des associations.

42 Cela a été souligné par Mireille Bonierbale.

43 Comme l'a indiqué Maud Thomas.

${ }^{44}$ C'est ce dernier choix théorique qui sous-tend la proposition de Tom Reucher de déplacer les « troubles de l'identité sexuelle » dans le chapitre 21 de la CIM («Facteurs influant sur l'état de santé et motifs de recours aux services de santé ». La stigmatisation fait partie de ces facteurs).

${ }^{45}$ Comme l'a soutenu Mireille Bonierbale.

46 Patrice Desmons.

47 Tom Reucher.

48 Alain Giami, Tom Reucher, Eric Macé.
}

qui rend impossible la déclassification complète ${ }^{49}$. Cette dernière $^{50}$ ne serait ni souhaitable, ni possible au niveau international, car elle serait synonyme de la perte d'accès aux soins, voire de la judiciarisation des trans' dans certains pays - l'argument valant aussi pour F65.

\section{Classification et identité sexuelle}

Le titre de la catégorie F64 engage directement les théories de l'identité sexuelle ${ }^{51}$. Les relations entre les identités subjectives et sociales et les catégories médicales ont ainsi été l'objet de bon nombre de réflexions au cours de cette journée ${ }^{52}$. Si les classifications sont performatives, elles produisent en effet des identifications dont elles constituent les points de repère - assumés ou refusés.

La présence sous-jacente ou explicite des théories constructivistes du genre ${ }^{53}$ a pesé de manière importante sur la formulation de l'ensemble des analyses, traversant le discours des chercheurs en sciences sociales et celui des associatifs. Ainsi, Éric Macé comme Maud Thomas ont insisté sur les singularités et la diversité des « devenirs trans' », des trajectoires d'identification de genre ${ }^{54}$, et des trajectoires d'existence, avec lesquelles les classifications actuelles seraient théoriquement inadéquates. Il faut cependant noter que cette conscience aigüe de la diversité des situations et des vécus trans' n'est pas l'apanage des chercheurs et sciences sociales et des associations, puisqu'elle a été soulignée par Colette Chiland ${ }^{55}$. Les efforts de déconstruction des modèles traditionnels essentialistes, binaires et hétérocentrés par les mouvements trans' ont ainsi été rappelés, ainsi que leurs effets généraux sur l'ensemble des identifications de genre, cisgenre compris. En parallèle, le retard théorique et juridique de la France sur ces questions a été pointé s6 $^{5}$

Un point de consensus imprévu semble ainsi avoir émergé à propos du protocole de réassignation. Pour des raisons tout à fait divergentes - lourdeur des conséquences médicales des procédures pour les psychiatres ${ }^{57}$, autonomie du choix d'un parcours de vie trans' singulier pour

\footnotetext{
49 Tom Reucher.

${ }^{50}$ Comme l'a rappelé Geoffrey Reed.

${ }^{51}$ Comme l'a bien montré Pierre-Henri Castel, la définition même que l'on peut donner de ces « troubles de l'identité sexuelle » dépend logiquement de la théorie de l'identité que l'on peut endosser. Castel, 2003, op. cit. 52 Éric Macé, Alain Giami, Maud Thomas.

53 Social et gender studies, mais aussi théories queer.

54 Eric Macé.

55 Maud Thomas a indiqué les implications pratiques de cette inadéquation : les équipes hospitalières françaises seraient incapables de faire face à la multiplicité des identités trans' parce qu'elles continueraient à prendre appui sur un modèle daté et binaire de la transsexualité (transition complète, avec réassignation chirurgicale).

56 Éric Macé, Maud Thomas. On peut à ce titre rappeler la traduction inadéquate de la catégorie F64 « Gender Identity Disorder » par « Troubles de l'identité sexuelle », qui témoigne de cette difficulté à dissocier sexe et genre dans l'espace français.

57 Colette Chiland.
} 
les associations - le parcours hormono-chirurgical complet de réassignation sexuelle a été problématisé. Si l'impératif juridique actuel en France de compléter ce parcours pour bénéficier d'un changement d'état civil n'a pas pu être traité dans le cadre de cette journée ${ }^{58}$, sa valeur de distorsion sur les débats et la vie des trans' ne peut être ignorée ${ }^{59}$.

L'ensemble des contributions est alors traversé par une tension, entre la particularité de la condition trans' et les exigences d'universalité. S'il a été souligné que l'idée de trajectoire d'existence pouvait s'appliquer généralement à l'ensemble des individus ${ }^{60}$, que le processus de déstigmatisation supposait la réintégration des questions trans' dans les questions plus générales des règles du droit commun ${ }^{61}$, la spécificité de la condition trans' a été en parallèle mise en avant ${ }^{62}$. Comment concilier la revendication de particularité avec la prise en compte de l'universalité, cruciale dans la perspective des droits humains, par définition universaliste, qu'adopte l'OMS ?

\section{Une question d'autonomie}

Les représentants des associations ont dénoncé la volonté de confiscation institutionnelle des psychiatres ${ }^{63}$, ce à quoi ces derniers ont répondu par la nécessité de l'accompagnement. L'opposition entre deux théories morales et politiques implicites du rapport à soi et à son corps sous-tend ce clivage. La première est une pensée du self-ownership dont découle l'autonomie, le droit à l'autodétermination, et la libre disposition de son corps, la seconde une conception qui met l'accent sur la nécessité de la protection et de la prise en charge par autrui, et souligne ainsi la dépendance des sujets à l'expertise et aux pratiques médicales. Ce clivage est apparu à de nombreuses reprises dans les discussions confrontant les représentants des associations et les psychiatres appartenant à la SoFECT : sur le choix du réseau médical, sur le statut de modèle de l'IVG dans la prise en charge du parcours de soin $^{64}$, sur la possible substitution d'une procédure de consentement éclairé à l'avis psychiatrique, sur la relation du sujet à son corps dans le parcours de réassignation - devant être indexé sur un choix éclairé pour les uns, dépendant d'une expertise $\mathrm{du}$ fait des graves implications biologiques et médicales pour les autres ${ }^{65}$. Chacune des deux conceptions soulève

\footnotetext{
58 Comme l'ont fait remarquer Colette Chiland et Tom Reucher.

59 Éric Macé a d'ailleurs souligné le retard juridique de la France sur ces questions.

60 Maud Thomas, Éric Macé.

61 Éric Macé.

62 Par Colette Chiland, mais aussi par Maud Thomas.

63 Plus particulièrement ceux de la SoFECT.

${ }^{64}$ Tom Reucher a proposé ce modèle, contesté par Colette Chiland ainsi par la SoFECT dans sa réponse au rapport de la Haute Autorité de santé. 65 Colette Chiland a ainsi souligné dans quelle mesure les procédures de réassignation transmuaient un organisme sain en organisme à traiter à vie, ainsi que le caractère mutilant des ablations d'organes.
}

des difficultés qui lui sont propres. Tandis que le point de vue des psychiatres maintient une limitation politiquement problématique au choix des sujets trans' et les déresponsabilise au moins partiellement, l'hyper-responsabilisation exigée par le principe d'autonomie ${ }^{66}$ conduit à interroger le destin des sujets plus fragiles, notamment les enfants et les adolescents ${ }^{67}$. On ne peut ignorer le poids des classifications et de leur fonction stigmatisante dans ce débat. La qualification de «malade mental» tend en effet à ôter au sujet ainsi qualifié et disqualifié une partie de son autonomie.

\section{Troubles de la préférence sexuelle, des problèmes spécifiques}

Les troubles de la préférence sexuelle ${ }^{68}$, ou «paraphilies », codés F65, ont été peu abordés. Ils posent des problèmes bien spécifiques ${ }^{69}$. La présentation de F65 a pris pour objet les cas d'agressions sexuelle judiciarisés (délinquance et criminalité sexuelles), ce qui laisse de côté nombre d'autres cas et crée un biais de sélection important aux effets théoriques conséquents. La polarisation des classifications sur les questions médico-judiciaires et leur articulation en France à l'obligation de soin pour les agresseurs sexuels, qui remet en question leur légitimité scientifique, clinique et nosographique, a été parfaitement soulignée. Cela conduit en effet à indexer les catégories sur des critères comportementaux et criminologiques, et non sur des critères psychologiques et psychopathologiques ${ }^{70}$, ce qui aboutit au maintien de catégories inadéquates et à la fabrication de groupes cliniques hétérogènes. Cette articulation étroite du psychiatrique et du judiciaire a conduit les intervenants à privilégier le cas de la pédophilie, classe hétérogène tendant à englober nombre de phénomènes bien distincts de pédocriminalité, comme exemple paradigmatique de la difficulté actuelle de F65 à constituer une classe cliniquement cohérente. Ces constats ont mené à énoncer la nécessaire révision de F65. Les implications thérapeutiques sont en effet lourdes : comment, dans le cadre de l'obligation de soin, élaborer une thérapeutique, si l'on ne sait pas vraiment qui soigner, ni ce que l'on doit soigner? Il faudrait cependant distinguer précisément les difficultés inhérentes aux catégories elles-mêmes, et celles qui découlent de leurs usages.

\footnotetext{
${ }^{66}$ Responsabilisation sur laquelle Tom Reucher n'a cessé de revenir. ${ }^{67}$ Ce problème a été soulevé par Pierre-Henri Castel au cours des discussions de milieu de journée.

68 Cette classe comprend les paraphilies du DSM : F65.0 Fétichisme, F65.1 Travestisme fétichiste, F65.2 Exhibitionnisme, F65.3 Voyeurisme, F65.4 Pédophilie, F65.5 Sadomasochisme, F65.6 Troubles multiples de la préférence sexuelle, F65.8 Autres troubles de la préférence sexuelle, F65.9 Trouble de la préférence sexuelle, sans précision.

${ }^{69}$ Ils ont été l'objet des interventions des psychiatres Tiphaine Séguret et Pierre Hum.

${ }^{70}$ Fantasmes ; souffrance individuelle ; évolutions des sujets.
} 
Le problème de la stigmatisation est aussi posé par F65, dans la mesure où les sujets catégorisés sont doublement stigmatisés, à la fois comme délinquants ou criminels condamnés et comme malades mentaux, ce dont l'usage actuel de «pédophilie », un des stigmates contemporains les plus forts, est le témoin.

Le problème posé par F65 est donc quadruple : premièrement, le caractère comportemental et non psychologique des critères actuels, les classifications étant indexées sur les actes et non sur les sujets ; le manque de représentativité nosographique et l'hétérogénéité des groupes constitués ; la stigmatisation consécutive à la catégorisation, notamment dans le cas de la pédophilie ; enfin, la place de la dimension sexuelle dans les cas censément visés par F65, peu évidente - Tiphaine Séguret soulignant ainsi la nécessité de distinguer entre le crime sexuel et le crime sexué, c'est-à-dire mettant en œuvre des moyens sexuels pour une finalité qui ne l'est pas.

L'idée cardinale est de dissocier crime et pathologie ${ }^{71}$, répression et soin, champ pénal et champ médical pour réviser entièrement la liste des troubles de la préférence sexuelle. Une déclassification pure et simple de $\mathrm{F} 65^{72}$ est difficilement envisageable, car elle conduirait à une sur-judiciarisation de certaines conduites délinquantes ou criminelles, dans un contexte déjà fortement sécuritaire.

\section{Perspectives critiques}

Quatre impensés de l'ensemble de cette journée peuvent être interrogés. Premièrement, en dépit des rapprochements avec les autres catégories de troubles mentaux qui ont été opérés, la volonté de dépsychiatrisation des identités trans' suppose l'acceptation implicite de l'infériorisation et de la stigmatisation des autres «malades mentaux », condition à laquelle les trans' souhaitent légitimement échapper. La possibilité, soulignée dans les échanges préalables à cette journée, d'un dépassement des logiques d'éclatement pour penser les discriminations d'un point de vue holistique, mériterait d'être repensée. Deuxièmement, l'absence de prise en compte de la diversité des sujets classés en F65 et celle d'une représentation des usagers concernés par les «troubles de la préférence sexuelle » sont problématiques. Les effets de stigmatisation des catégories comme «fétichisme » ou « sado-masochisme » peuvent en effet être questionnés. Troisièmement, la place du sexuel est restée quasiment vide pour l'ensemble des analyses. Celles portant sur «les troubles de l'identité sexuelle» ont globalement insisté sur la nécessaire désarticulation de l'identité de genre et de la sexualité. La volonté de se diriger vers un paradigme de la santé sexuelle plutôt que de la santé mentale, qui rapproche alors les questions trans' des

\footnotetext{
71 Jean-Luc Roelandt.

72 Suggérée par Geoffrey Reed.
}

problèmes liés à la santé reproductive $\mathrm{e}^{73}$ renforce cette difficulté. L'érection de la souffrance individuelle en norme de la « vraie » transsexualité par les médecins a été critiquée par les associations ; mais l'éviction complète du plaisir et de la sexualité des débats ${ }^{74}$ ne peut qu'interroger. La même question peut être adressée au traitement de F65, face au silence sur les sexualités catégorisées comme psychopathologiques mais non délinquantes ou criminelles.

Quatrièmement, l'usage et le statut des différents paradigmes invoqués mériteraient clarification, car ils sont sujets à débats entre psychiatres et associations. On pensera notamment à l'homosexualité, dont le statut est, pour les associations, celui d'un modèle historique et politique ${ }^{75}$, et non épistémologique comme le suggèrent les psychiatres, à l'IVG, invoquée comme un modèle pratique et administratif par les associations ${ }^{76}$, et non théorique ou biologique, et enfin aux relations entre trans' et intersexes, équivoques, puisque leurs rapprochements ${ }^{77}$ et la mise en évidence de leurs différences ${ }^{78}$ sont alternativement soulignés. Pour les troubles de la préférence sexuelle, l'usage paradigmatique de la pédophilie est lui aussi à analyser : dans quelle mesure est-elle un modèle adéquat pour repenser les autres catégories ?

\section{Résumé des propositions de transformations des classifications}

\section{Impératifs et cadre}

L'intervention de Geoffrey Reed a permis de rappeler les attendus de l'OMS pour l'élaboration de la CIM-11. Les impératifs pratiques qui tracent les limites du cadre de

\footnotetext{
${ }^{73}$ Rapprochement institutionnellement acté par la prise en charge de la révision de F64 par le groupe de «Santé sexuelle et reproductive » de l'OMS à Genève. Si Geoffrey Reed a expliqué qu'il s'agissait d'un choix stratégique, il a néanmoins souligné les difficultés que ce rapprochement posait des difficultés.

74 Alors qu'ils appartiennent à l'ensemble des droits sexuels revendiquée et émergeant à l'échelle internationale depuis le milieu des années 1990.

${ }^{75}$ Les luttes homosexuelles ont mené au retrait de l'homosexualité des classifications du second Diagnostic and Statistical Manual of Mental Disorders (DSM-II) de l'American Psychiatric Association (APA) en 1974. L'homosexualité était codée 302.0 (classe des « déviations sexuelles ») dans le chapitre V du DSM-II (APA. DSM-II. Diagnostic and Statistical Manual of Mental Disorders [second edition], Washington DC : American Psychiatric Association, 1968, p. 10-44). La recommandation de retrait fut émise en 1973, et le vote majoritaire de l'APA l'adoptant en 1974 (APA. Homosexuality and Sexual Orientation Disturbance: Proposed Change in DSM-II [6th Printing]. Arligton : APA, 1974, p. 44. <www.psychiatryonline.com/DSMPDF/DSMII_Homosexuality_Revision.pdf $>$ ). Néanmoins, son remplacement dans le DSM-II par la catégorie "Sexual Orientation Disturbance », puis l'introduction dans le DSM III de 1980 de l' « Ego-dystonic homosexuality » (qui ne disparut qu'en 1987, dans la révision du DSM-III ayant donné lieu au DSM III-R), furent aussi l'objet de controverses.

76 Tom Reucher.

77 Vincent Guillot.

78 Tom Reucher.
} 
la révision des classifications sont les suivants : premièrement, la possibilité d'une prise en charge économique des soins pour les trans', non seulement en France, mais aussi au niveau international, constitue un facteur essentiel dans la révision de la classification, et fait obstacle à une pure et simple déclassification. Il est, sur cette question, nécessaire d'endosser un point de vue pragmatique ${ }^{79}$ et de proposer, pour le cas français, une catégorie qui autorise le maintien dans la liste des ALD (donnant droit à un remboursement à $100 \%$ des soins). La dépsychiatrisation ne peut donc être synonyme de dépathologisation. La création d'un nouveau chapitre est possible. Deuxièmement, la valeur internationale de la CIM oblige à prendre en compte la variabilité transnationale des politiques de santé et du traitement des troubles du genre. Il faut donc élaborer les propositions à la lumière du mandat mondial de $\mathrm{l}^{\prime} \mathrm{OMS}^{80}$. Troisièmement, la prise en compte des effets de stigmatisation, et des droits humains, est incontournable. Le rapport Hammarberg constitue à ce titre une référence ${ }^{81}$. Enfin, si chacun peut élaborer une proposition, le travail en collaboration des associations-usagers et des professionnels de la santé mentale débouchant sur des propositions fortes est préférable. Dans tous les cas, le point de vue des usagers est lui aussi incontournable.

\section{Propositions de révision de F64}

Avant les discussions, trois propositions ont été émises par Colette Chiland, Tom Reucher et Maud Thomas. On trouvera leur détail dans les textes des trois auteurs. Colette Chiland a proposé un réaménagement de F64, Tom Reucher a formulé plusieurs propositions visant à intégrer la transidentité dans le chapitre $21^{82}$ - la difficulté étant que la catégorie $\mathrm{Z}$ ne permet pas de fonder le remboursement des soins par l'Assurance maladie ${ }^{83}$ - et Maud Thomas a proposé de conserver une notation dans F 60 « Troubles spécifiques de la personnalité » tout en insistant sur une nécessaire prise en charge psychologique globale, le choix du médecin devant rester imprescriptible.

Après discussions, les directions de travail suivantes ont été proposées. Tom Reucher a résumé les souhaits des usagers : une dépsychiatrisation des identités trans', sans démédicalisation, en conservant la possibilité d'une

\footnotetext{
79 Tom Reucher.

80 Geoffrey Reed.

81 Geoffrey Reed.

82 «Facteurs influant sur l'état de santé et motifs de recours aux services de santé », entre Z00 et Z76. L'accent était mis sur les troubles consécutifs à la stigmatisation.

83 Comme l'ont souligné plusieurs intervenants au cours de la journée, notamment Jean-Luc Roelandt et Tiphaine Séguret lors des discussions du midi.
}

prise en charge complète du parcours de soins. Le point de vue des psychiatres s'est exprimé doublement. La présidente de la SoFECT a insisté sur la nécessité d'un étayage scientifique des propositions, tandis que Colette Chiland a proposé la création d'un chapitre qui serait générateur de remboursement des soins pour sortir de l'alternative F/Z. Il semblerait donc qu' un consensus partiel, mais pour l'instant purement formel, ait émergé : l'abandon du modèle ancien de la transsexualité, et la possibilité de créer un nouveau chapitre qui réponde aux attendus théoriques, médicaux, et économiques des questions trans', en tenant en compte de la variabilité des politiques de santé internationale, et des droits humains.

\section{Propositions de révision de F65}

Il est nécessaire de réélaborer les critères afin d'éviter la confusion entre crime et pathologie, et de réviser la liste de F65 ${ }^{84}$. Trois possibilités sont ouvertes :

- un changement du titre de F65, car la place de la sexualité n'est pas évidente cliniquement. L'alternative « troubles du comportement et des fantasmes sexuels » a été proposée ; - le démembrement partiel de F65 pour redistribuer ses éléments dans d'autres classes psychopathologiques (les troubles des impulsions et les troubles obsessionnels ont été évoqués) ;

- la transformation du contenu : réunion en une même catégorie des diagnostics rares ; classification des pédophiles, avec un travail sur les critères ; apparition du viol d'adulte.

Conflits d'intérêts : aucun.

\section{Bibliographie}

APA. Homosexuality and Sexual Orientation Disturbance: Proposed Change in DSM-II, 6th Printing. Position statement (Retired). Arligton (VA) : APA, document reference No. 730008, 1974, 5 p. <www.psychiatryonline.com/DSMPDF/DSM-II_ Homosexuality_Revision.pdf $>$.

APA DSM II. Diagnostic and Statistical Manual of Mental Disorders (second edition), Washington DC : APA, 1968, 119 p.

Hammarberg T. Human Rights and Gender Identity. Strasbourg : Office of the commissionner of human rights, Council of Europe, 29 Juillet 2009, 53 p. <https://wcd.coe.int/wcd/com. instranet.InstraServlet?Index $=$ no\& command $=$ com.instranet. CmdBlobGet\&InstranetImage $=1670860 \&$ SecMode $=1 \&$ DocId $=1433126 \&$ Usage $=2>$ et $<$ https: $/ /$ wcd.coe.int $/ \mathrm{wcd} /$ com.instranet.InstraServlet?Index $=$ no $\&$ command $=$ com. instranet.CmdBlobGet\&InstranetImage $=1692292 \&$ SecMode $=1 \&$ DocId $=1458356 \&$ Usage $=2>$.

Castel PH. La Métamorphose impensable. Essai sur le transsexualisme et l'identité personnelle. Paris : Gallimard, 2003, 551 p.

${ }^{84}$ Jean-Luc Roelandt, Tiphaine Séguret, Pierre Hum. 